\title{
LOS PARÁMETROS QUE IDENTIFICAN EL SUBTITULADO PARA SORDOS. ANÁLISIS Y CLASIFICACIÓN ${ }^{1}$
}

\author{
Verónica Arnáiz Uzquiza \\ Universidad de Valladolid (España) \\ Universitat Autònoma de Barcelona (España) \\ v_arnaiz@hotmail.com
}

\section{Resumen}

En los últimos años ha aumentado la presión social a favor de la accesibilidad audiovisual, lo que ha venido acompañado de un incremento en el número de productos subtitulados para sordos disponibles en el mercado. El significativo crecimiento en el número de estudios ha cuestionado la aplicación sistemática de los parámetros generales del subtitulado a la práctica del Subtitulado para Sordos (SPS). Partiendo de una propuesta de Bartoll (2008) para el estudio de los parámetros del subtitulado, el presente artículo plantea la adaptación de la taxonomía al estudio del SPS. Analizando los parámetros de naturaleza general aplicables al SPS, incorporando aquellos parámetros exclusivos de esta modalidad (la representación de la información extralingüística), y considerando las interconexiones que se establecen entre todos ellos, la nueva taxonomía busca servir en el estudio integral y detallado de cada uno de los aspectos que configuran la práctica del SPS.

\begin{abstract}
"A taxonomy on the parameters of subtitles for the Deaf and Hard-of-Hearing. Analysis and classification"

Subtitling for the Deaf and the Hard-of-Hearing (SDH) was long considered a "simple" variant of standard subtitling. Only recently, uprising social demands together with a growing presence of SDH materials in different international audiovisual environments, have promoted the proliferation of research initiatives on SDH practices. As a result, the systematic application to SDH of some of the parameters originally adopted for standard subtitling has proven to be controversial. This paper presents a proposal for the specific analysis of SDH parameters. Based on a taxonomy developed
\end{abstract}


by Bartoll (2008), the new taxonomy describes the restricted application of standard parameters to this accessibility modality. The new proposal focuses on the parameters that are specific to SDH -the representation of extralinguistic information- and sheds light into the tight connection established among all the agents involved. The new taxonomy tries to provide researchers and SDH professionals with a tool to evaluate SDH practices and analyze the implications of potential modifications on parameters.

Palabras clave: Subtitulado para Sordos (SPS). Taxonomía. Parámetros. Análisis. Información extralingüística.

Keywords: Subtitling for the Deaf (SDH). Taxonomy. Parameters. Analysis. Extralinguistic information.

Manuscript received on July 7, 2011; Definitely accepted on November 15, 2011.

1. Esta investigación ha sido posible gracias al proyecto "La subtitulación para sordos y la audiodescripción: pruebas objetivas y planes de futuro" (2009-2012), financiado por el Ministerio de Ciencia e Innovación de España (FFI2009-08027), y a los fondos de la Generalitat Catalana 2009SGR700. 


\section{Introducción}

A lo largo de la última década se ha producido un significativo incremento en la presencia del Subtitulado para Sordos (SPS) en el contexto audiovisual internacional. La creciente presión social, unida a una mayor flexibilidad de medios y soportes, ha incentivado la producción y distribución de esta herramienta de accesibilidad. Es precisamente la mayor visibilidad de la práctica y su creciente profesionalización la que ha dado origen en los últimos años a un cada vez mayor número de estudios sobre la cuestión, surgidos desde los más diversos ámbitos de especialidad, desde la Ingeniería (cf. Martín et al. 2007 y 2008), a la Psicología (cf. Cambra et al. 2008), pasando por los Estudios de la Sordera (cf. Jensema et al. 2000) o el Derecho (cf. Pérez-Ugena et al. 2010). En este sentido, resultan de especial interés los promovidos desde los Estudios de Traducción, por su visión transversal y el carácter interdisciplinar del que suelen estar dotados.

Si bien es cierto que el SPS es, a menudo, considerado una de las muchas disciplinas que integran la práctica del subtitulado ordinario, son precisamente sus características diferenciales las que invitan a una clasificación específica. Como recoge De Linde (1996: 182):

Le sous-titrage intralinguistique souligne de fait les interrelations subtiles entre parole, écrit et visuel, en tentant de reproduire l'information sonore par d'autres éléments qu'auditifs tout en cherchant à garder un certain équilibre spatio-temporel avec les images.

El contenido sonoro adicional de la obra audiovisual que es preciso representar mediante el SPS, como señala De Linde, hace necesario incorporar parámetros de estudio exclusivos o de especial interés dentro de esta modalidad. Centrándose principalmente en elementos lingüísticos, pero abordando también cuestiones formales o técnicas, diversos autores se han adentrado en el estudio del SPS desde el ámbito traductológico (cf. De Linde 1996; De Linde y Kay 1999; Neves 2005; Pereira 2005, entre otros). Sin embargo, el elevado número de elementos que configuran el desarrollo de esta especialidad de subtitulado sigue haciendo necesaria una clasificación que permita describir cada uno de los aspectos que dan forma al producto final. 


\section{Taxonomía del subtitulado de Bartoll (2008)}

A pesar de que, hasta la fecha, no se ha llevado a cabo en el ámbito del SPS ninguna propuesta de análisis taxonómico, sí que existen iniciativas surgidas para el estudio del subtitulado ordinario. Basándose en estudios previos de Gottlieb (1997), Ivarsson y Carroll (1998) y Karamitroglou (1998), Bartoll (2008) ha elaborado una propuesta con el objeto de establecer un modelo de análisis y clasificación de los parámetros del subtitulado que permita determinar los diferentes tipos de subtitulado que se desarrollan en el mercado. El autor identifica hasta 15 parámetros, organizados en torno a tres perfiles, como aspectos configuradores del producto subtitulado:

- Parámetros lingüísticos: entre los que recoge los parámetros "Lengua" y "Densidad".

- Parámetros pragmáticos: con aspectos como "Destinatarios", "Intención", "Tiempos de Elaboración" y "Autoría".

- Parámetros técnicos: recoge elementos como "Opcionalidad", "Difusión", "Color", "Incorporación", "Posicionamiento", "Emplazamiento", "Archivado", "Tipografía" y "Formato".

El detallado conjunto de parámetros que el autor logra recopilar, y la relación de interdependencia que refleja entre ellos, resultan pioneros a la hora de representar la compleja estructura que tiene lugar en el proceso de elaboración de subtítulos. Como desvela el autor, la elección de una determinada variable en uno de los parámetros supone la alteración de la variable en otro diferente, produciendo un efecto en cadena que quedará reflejado en el aspecto final del subtítulo.

Si bien en su detallada propuesta estaría incluido el SPS, el mismo autor recoge la posible revisión de su modelo en función de las nuevas aportaciones que surjan en materia de accesibilidad a los medios (ibid: 4). Por este motivo, en el intento de aplicación de su modelo al análisis del SPS, se plantea la necesidad de ampliar su propuesta.

El análisis de estudios específicos sobre la práctica del SPS, como el de Neves (2005), y de algunas de las normativas públicas de SPS disponibles (cf. AENOR 2003; BBC 2009; BCI 2005; CAB 2008; DFA 2004; DCMP 2011; ITC 1999) desvelan la existencia de una serie de elementos específicos del SPS, condicionados fundamentalmente por la representación de la información sonora adicional, que no aparecen representados en el trabajo de Bartoll. Su incorporación, y efecto sobre otros parámetros, dan origen a la nueva propuesta que aquí se recoge. 


\section{Taxonomía del SPS}

Prestando especial atención a los elementos específicos del SPS, como es el caso de los elementos sonoros externos al diálogo, y a aquellos elementos que desempeñan una función significativa en el desarrollo de los productos subtitulados para sordos, tales como la velocidad de los subtítulos y la tipografía, con este trabajo proponemos la generación de una nueva taxonomía. El nuevo modelo recoge dos de las categorías ya propuestas por Bartoll ("Parámetros lingüísticos" y "Parámetros pragmáticos") y modifica la tercera categoría, "Parámetros técnicos", al considerar que los elementos que la integran pueden agruparse en tres categorías diferenciadas: "Parámetros estéticos", "Parámetros técnicos" y "Parámetros estético-técnicos". Estos últimos hacen referencia a un grupo de parámetros cuyo resultado estético no depende de la elección del subtitulador, sino que viene impuesto por el proceso de producción (cf. apartado 2.6.). Pero la principal aportación a esta nueva taxonomía es, posiblemente, la incorporación de una sexta categoría, "Parámetros extralingüísticos sonoros", diseñada para el análisis específico de los elementos sonoros recogidos de forma exclusiva en SPS.

A las nuevas aportaciones clasificatorias hay que añadirle la incorporación de nuevos parámetros en algunas de las categorías, como son "Justificación", "Método de elaboración" y "Velocidad", así como la inclusión de nuevas variables en algunos de los parámetros ya existentes (cf. anexo I).

\subsection{Parámetros lingüísticos}

Los "Parámetros lingüísticos" planteados por Bartoll, "Lengua" y "Densidad", mantienen su vigencia en el estudio del SPS, aunque con un enfoque específico. A pesar de que autores como De Linde (1996) o De Linde y Kay (1999) equiparaban la subtitulación intralingüística al SPS, Díaz-Cintas recogió otras cuatro modalidades diferentes de subtitulación intralingüística, al margen de esta: para personas con déficit auditivo, para el aprendizaje de idiomas, efecto karaoke, variantes del mismo idioma y noticias y publicidad (2003: 38). Cabe destacar aquí que la consideración lingüística (parámetro "Lengua") del SPS no depende únicamente de las lenguas origen y meta inherentes a la traducción, sino que viene marcada por la tradición audiovisual del contexto de llegada. Mientras en países dobladores, como España, el SPS representa una actividad de naturaleza mayoritariamente intralingüística, en países de tradición subtituladora la presencia original de subtítulos dificultó en sus comienzos el desarrollo de una modalidad específica para sordos por considerarla innecesaria (cf. De Linde y Kay 1999: 8). Afortunadamente, la 
eclosión de los nuevos soportes y de la conciencia social ha justificado el desarrollo de esta modalidad, al margen de la lengua y de la tradición audiovisual (cf. Neves 2005: 241; Neves 2009: 152). Por este motivo, fruto de este contexto audiovisual heterogéneo, salvo en contadas excepciones, no resulta habitual encontrar referencia alguna al parámetro "Lengua" en el estudio del SPS, ni en ninguna de las normativas publicadas que regulan su producción. Arnáiz-Uzquiza (2007) señala que el ejercicio del SPS se basa en una serie de normativas cuya difusión suele estar limitada al contexto privado. Son escasos los ejemplos públicos disponibles, en su mayoría procedentes de instituciones públicas o privadas de países de lengua inglesa (cf. apartado 2).

El parámetro "Densidad", que recoge la relación entre la cantidad de información textual presentada en el subtítulo y la información verbal procedente de la pista sonora, está sujeto a otro tipo de enfoque. Además de estar supeditado a las características del producto audiovisual y a las restricciones espacio-temporales propias del subtitulado, pueden ser varios los condicionantes impuestos que limiten, y se vean limitados por este parámetro, como es el caso del número de caracteres por línea, el número de líneas, o la velocidad de lectura, entre otros. Las restricciones espacio-temporales que se aplican al subtitulado ordinario ya revelan la imposibilidad (actual) de llevar a cabo una transcripción literal del subtítulo y, dependiendo de las lenguas de trabajo, apuntan a unas tasas de reducción que pueden oscilar entre el 22\% y el 75\% del texto original (cf. Lonheim 1995: 203; Lorenzo 2001: 15; DíazCintas 2003: 202; Gottlieb 2005: 20). No obstante, en lo que al SPS se refiere, resulta habitual la demanda por parte del público con deficiencia auditiva de una transcripción literal de los diálogos (cf. Ofcom 2005: 16; RomeroFresco, en prensa). Estas exigencias, cuya justificación radicaría no solo en el desconocimiento de la técnica, sino también en el deseo de disponer de toda la información ofrecida en el original, aparecen reflejadas en la apuesta por la literalidad de los textos normativos consultados. A pesar de que la evolución médica y sociocultural en el seno de la comunidad con problemas de audición hace que sea posible hablar de una mejora en los niveles de alfabetización, y con ello, de una mejora en las capacidades lectoras de los usuarios de SPS con respecto a generaciones anteriores, la heterogeneidad de la comunidad sorda revela que las capacidades lectoras de parte de este grupo de usuarios no aconsejan la transcripción literal si lo que se pretende es garantizar la accesibilidad al contenido. Los estudios desarrollados por Cambra et al. (2008), Lorenzo (2010a), Pereira (2010), Romero-Fresco (en prensa) o Zárate (2010) en fechas recientes muestran los problemas de algunos usuarios con deficiencia auditiva para comprender el SPS actual, especialmente en el caso de 
aquellos usuarios cuya primera lengua es la Lengua de Señas (LS). Al mismo tiempo hay que tener en cuenta que el SPS se caracteriza, entre otros rasgos, por incorporar información extralingüística al subtitulado ordinario, lo que supone un incremento en el número total de caracteres que el espectador debe leer. Conscientes de esta situación, algunos textos normativos recogen la posibilidad de desarrollar otras modalidades de subtitulado -editado, o reducido simplificado- para públicos específicos, como el público infantil (cf. BBC 2009: 30), los niños con sordera prelocutiva (cf. ITC 1999: 19; BCI 2005: 10) o el público con problemas de lectoescritura. A este respecto, la norma española no determina el perfil del público con problemas de lectoescritura (cf. AENOR 2003: 12).

No obstante, determinados usuarios se muestran reticentes ante la reformulación del subtítulo con el fin de dotarlo de características lingüísticas más idóneas para los espectadores signantes (cf. Lorenzo 2010a: 121; Pereira 2010: 100). Para ellos, el colectivo de usuarios signantes únicamente tendría sus necesidades comunicativas cubiertas con la ayuda de intérpretes de LS y no mediante el SPS, como recogen De Linde y Kay (1999: 10), siguiendo los estudios de Woll (1991).

\subsection{Parámetros extralingüísticos sonoros}

Siguiendo la clasificación de Neves (2005: 220-258), los parámetros extralingüísticos sonoros constituyen el principal punto identificativo del SPS y suponen la principal incorporación a la propuesta de Bartoll (2008). Aunque este grupo de parámetros supone uno de los aspectos recogidos con más frecuencia por las normativas sobre SPS, su presencia aparece reflejada de forma desigual en la mayoría de los casos.

Esta categoría hace referencia a la representación de toda la información sonora de índole no verbal que forma parte del documento audiovisual. La naturaleza no verbal de esta información hace que, ante la ausencia de un referente visual de acompañamiento, sea preciso representarla por escrito para que el espectador con problemas de audición pueda alcanzar unos niveles de comprensión equiparables a los del público normo-oyente. La diversidad de las fuentes y tipos de información sonora hace que resulte compleja la elección de un único término para la definición de este grupo de parámetros. El DRAE (2011) recoge que el término "extralingüístico" hace referencia a "todo elemento externo a la lengua que ayuda a la desambiguación de palabras y frases". De este modo se podría definir determinados elementos acústicos que acompañan al componente verbal en la obra audiovisual y que sirven para contextualizarlo, como son los efectos sonoros y la música. Sin embargo, 
existen otros dos elementos, la información paralingüística y la identificación de personajes, que, sin ser totalmente externos a la lengua, también pueden cumplir funciones de desambiguación basándose en su componente acústico, por lo que se ha considerado pertinente su incorporación dentro de esta categoría de "Parámetros extralingüísticos sonoros".

El primero de los parámetros de este grupo, "Información paralingüística", aporta carga propia en el proceso de lectura y comprensión, ya que su función es la de ampliar y esclarecer los parlamentos de los personajes. Es habitual su consideración en la gran mayoría de las normativas de SPS en conjunto con el parámetro "Efectos sonoros", como parte de un único parámetro que hace referencia a la representación de la información sonora. Sin embargo, el tipo de información al que ambos parámetros hacen referencia, como sugieren Neves (2005: 220) y Pereira y Lorenzo (2005: 24), aconseja una clasificación independiente de ambos componentes. Esta información paralingüística, que ambas autoras recogen como "rasgos paralingüísticos" y "didascalias" respectivamente, se correspondería con la "Información paralingüística" aquí propuesta y representaría aquellos matices de la interpretación de los personajes que, por no contar con un referente visual, únicamente dependen de su naturaleza acústica, como son los aspectos calificadores o diferenciadores de la voz (cf. Poyatos 1994b). Su representación práctica mediante la descripción suele ser generalizada, si bien es habitual su uso combinado con otras modalidades, como son el empleo de emoticonos para la información de naturaleza emocional (cf. AENOR 2003: 15); la representación cromática de los parlamentos (cf. Bouzinac 2008: 5); o la representación ortotipográfica (cf. AENOR 2003: 14; BBC 2009: 26), siendo esta última la de uso más extendido.

El segundo de los "Parámetros extralingüísticos sonoros" incorporados en esta categoría, "Identificación de personajes", es uno de los elementos más representativos del SPS debido a su visibilidad y peso específico en los textos académicos y prácticos sobre SPS. A pesar de que no se suele profundizar en su componente acústico, según Poyatos la identificación de un personaje tiene lugar a través del desciframiento de las cualidades primarias de la voz (Poyatos 1994b: 25-80). Recogida por Neves (2005: 236) como una forma de "localización, descripción y ubicación de la voz humana", el parámetro "Identificación de personajes" aporta información que permite al espectador asociar los diálogos escritos a cada uno de los personajes en pantalla, visibles o no. A pesar de que, por su análisis de las cualidades de la voz, este aspecto podría formar parte del paralenguaje, por lo que sería posible encuadrarlo dentro del parámetro anterior, el tipo y relevancia específica de la información que representa invita a su análisis como un parámetro independiente, dejando 
el parámetro "Información paralingüística" para la descripción de reacciones fisiológicas y emocionales.

Son varias las técnicas que se pueden emplear para la "Identificación de personajes" (cf. De Linde y Kay 1999: 15), y que, a menudo, condicionan la elección de variables entre los parámetros estéticos del subtítulo. Una de las técnicas más extendidas, al ser una de las que menos condicionan la configuración estética, pues añade únicamente rasgos cromáticos al texto, es la asignación de colores a cada uno de los personajes. A pesar de ser una de las técnicas que menor esfuerzo cognitivo requiere por parte del espectador (cf. King et al. 1994: 332), cabe destacar los conflictos que pueden plantearse en la representación de un elevado número de personajes (cf. Pereira y Lorenzo 2005: 11) y la limitada oferta cromática, marcada por las restricciones tecnológicas de los sistemas de emisión (cf. King et al. 1994: 333; AENOR 2003: $5)^{1}$. La segunda técnica de identificación de uso más extendido es el desplazamiento lateral o vertical del texto para situarlo cerca del personaje, empleada principalmente en Estados Unidos y Canadá (cf. DCMP 2001: 19; CAB 2008: 18). Aunque en la mayoría de los países se opta por aplicar la identificación cromática o el desplazamiento de forma exclusiva, en algunas ocasiones se plantea el uso de estas opciones de forma simultánea (cf. Neves 2005: 242).

Otras técnicas de identificación de uso generalizado son el empleo de etiquetas, a modo de acotaciones, precediendo al subtítulo cuando no es posible identificar la intervención del personaje en pantalla (cf. AENOR 2003: 16; CAB 2008: 18; DCMP 2011: 19) o el uso de puntuación distintiva, como guiones o comillas latinas (cf. BBC 2009: 15-16). Las nuevas posibilidades tecnológicas en el mercado y los perfiles de los usuarios finales (cf. RomeroFresco, en prensa) hacen que en los últimos años se estén planteando nuevas alternativas, como la incorporación de avatares para la identificación de personajes (cf. Quoc y Fels 2009) o el uso combinado de color y etiquetas como acompañamiento al texto monocromo (cf. Quoc y Fels 2010). Sin embargo, se sigue analizando la aceptación por parte del usuario final de cada una de estas opciones.

El tercero de los "Parámetros extralingüísticos sonoros", "Efectos sonoros", recoge toda la información kinésica sonora (cf. Poyatos 1994a) de naturaleza no paralingüística ni musical que tiene lugar dentro de la obra audiovisual y que afecta al desarrollo de la misma. Este tipo de información,

1. A pesar de que el teletexto analógico ha sido reemplazado, o se encuentra en proceso de reemplazamiento, por su versión digital en la mayoría de los países europeos, los aspectos cromáticos se siguen manteniendo debido a cuestiones de legibilidad (cf. BBC 2009: 38; AENOR, en prensa). 
que habitualmente no se representa en el subtitulado ordinario, debe recogerse en el SPS para que el espectador con problemas de audición alcance la máxima comprensión del producto audiovisual. De todos los "Parámetros extralingüísticos sonoros", son precisamente "Efectos sonoros" e "Identificación de personajes" los parámetros más demandados por parte del público con discapacidad auditiva (cf. Romero-Fresco, en prensa), de modo que no sólo aparecen recogidos en todos los estudios surgidos desde el ámbito académico (cf. Neves 2005: 243; Lorenzo 2010a: 126; Lorenzo 2010b: 137; Civera y Orero 2010: 152; Pereira 2010: 89), sino también en las guías de estilo y en las normativas surgidas desde el entorno profesional (cf. BCI 2005: 6; BBC 2009: 17) o los entes asesores y reguladores (cf. ITC 1999: 13; AENOR 2003: 6; DFA 2004: 5; CAB 2008: 18; DCMP 2011: 17). Aunque la representación de la información recogida por "Efectos sonoros" suele producirse en la práctica totalidad de los casos por medio de una descripción, los parámetros estéticos aplicados en su representación (posicionamiento y, especialmente, tipografía) difieren sensiblemente de unos países a otros (cf. Neves 2005: 243; RomeroFresco, en prensa). Del mismo modo, las pautas de redacción del componente lingüístico de esta información no suelen tener reflejo en las diferentes normativas, por lo que es frecuente la falta de consistencia en este sentido (cf. Arnáiz-Uzquiza 2007). Siguiendo la estela de la representación icónica de la información paralingüística, en los últimos tiempos han surgido iniciativas que plantearían la representación icónica de los efectos sonoros (cf. Civera y Orero 2010: 152). No obstante, la desigual acogida por parte de los usuarios arroja resultados contradictorios en función de su edad y grado de exposición a otros medios audiovisuales, por lo que, tal y como sucede con la identificación de personajes, esta, y otras opciones, continúan siendo objeto de estudio (cf. Romero-Fresco, en prensa).

El último de los parámetros que configuran este grupo es "Música". Aunque también en este caso suele ser habitual su catalogación dentro del parámetro "Efectos sonoros", el papel que desempeña en la obra audiovisual va mucho más allá. A pesar de que son pocos los autores que han abordado su estudio (cf. Neves 2005: 252; Pereira 2005: 24; Weber 2010: 31), su compleja naturaleza, diegética, como parte visual integrante de la obra musical, o extradiegética, que sin estar presente en la trama sirve para configurar la realidad audiovisual, hace que resulte determinante su análisis como un parámetro independiente. Muchas normativas, como es el caso de la UNE-153010, abordan 
este parámetro de forma meramente tangencial ${ }^{2}$ ya sea desde el punto de vista estético, lingüístico o extralingüístico. Sin embargo, en las guías de estilo más recientes se le presta una atención especial, llegando a indicar determinados aspectos lingüísticos (cf. CAB 2008: 7), o incluso pautas detalladas sobre la forma de subtitular estos elementos atendiendo a su relevancia en la escena, carácter, etc. (cf. BBC 2009: 31).

\subsection{Parámetros pragmáticos}

Los "Parámetros pragmáticos" definidos por Bartoll son una de las categorías que experimentan menos modificaciones en la adaptación de la taxonomía al análisis del SPS, al no contar con ninguna nueva incorporación. Desde el punto de vista práctico, son escasas las referencias a este grupo de parámetros en estudios y textos normativos; sin embargo, la presencia de los "Parámetros extralingüísticos sonoros" condicionaría, y se vería condicionada, por las variables pragmáticas aplicables al margen de los rasgos específicos del SPS.

Mientras aspectos como "Autoría" siguen haciendo referencia al 'agente', humano o no, que desarrolla el SPS, el "Momento de elaboración", que también en SPS se limita a las variables "Anteriores" y "Simultáneos", condiciona la posibilidad de incluir información extralingüística dada la dificultad para recoger esta información de forma sincronizada (cf. Romero-Fresco 2011: 38). Por este motivo en los últimos cinco años, con la necesidad de proveer de accesibilidad audiovisual a eventos en directo, se ha incluido en la redacción de algunas normativas breves referencias a este parámetro (cf. BCI 2005: 11; CAB 2008: 22; DCMP 2011: 4; DFA 2004: 4).

Algo similar sucedería con el parámetro "Intención", que aborda el objetivo que se persigue con los subtítulos. Una vez más, y sin ser exclusivo del SPS, resulta importante la incorporación de una nueva variable en este parámetro, como son los "Subtítulos Terapéuticos", desarrollados para abordar problemas específicos de aprendizaje, y que, dependen, en gran medida, de uno de los parámetros esenciales en esta categoría: "Destinatarios".

El último de los parámetros de esta clasificación, "Destinatarios", aparece recogido por Bartoll para abordar la diferente naturaleza de dos grandes grupos de usuarios, sordos y oyentes. Sin embargo, son otros muchos los aspectos que es preciso tener en consideración a la hora de definir el perfil del usuario. Aspectos como la edad, relevantes para el desarrollo de todo tipo

2. La norma UNE-153010 (AENOR 2003: 14) únicamente recoge en su texto: "Se debe subtitular las canciones, en cuyo caso debe realizarse sobre fondo amarillo con carácter azul". 
de subtítulos, interactúan con cuestiones específicas de los usuarios de SPS, como son el perfil lingüístico (signante / oralista), el tipo, grado y momento de aparición de la sordera, o las necesidades terapéuticas de los usuarios (cf. Llombart 2007). Todas estas diferencias marcarían, por ejemplo, la capacidad del espectador para acceder a la información del componente sonoro, una mayor o menor velocidad de lectura o la familiaridad con el lenguaje escrito. No obstante, a pesar de que todas las normativas escritas consultadas se elaboran para personas con problemas de audición, teniendo en cuenta la diferente etiología de los grupos de usuarios, cabe destacar que en contadas ocasiones se hace referencia en los textos al perfil específico del usuario final. Por el contrario, las variables adoptadas en todos los casos van encaminadas a un perfil de usuario determinado: usuarios oralistas con índices de alfabetización medios-altos y destrezas lectoras elevadas.

\subsection{Parámetros estéticos}

Bartoll (2008: 260) define un único grupo de parámetros, "Parámetros técnicos", para hacer referencia a todos los aspectos relacionados con la parte visual y técnica del proceso de elaboración del subtítulo. Dada la variedad de los aspectos incorporados en esta categoría, sería posible identificar hasta tres grupos diferentes de parámetros en esta categoría: "Parámetros estéticos", "Parámetros estético-técnicos" y "Parámetros técnicos".

El primero de todos, "Parámetros estéticos", agruparía todos los elementos planteados por Bartoll que marcarían el aspecto más visual del subtítulo, y cuya elección puede variar por elección del subtitulador, tales como "Emplazamiento", "Color", "Tipografía" y "Posición”. Cabe destacar la incorporación de un nuevo parámetro, "Justificación", que, al margen del parámetro "Posición", recoge la disposición de subtítulo con respecto a un margen establecido.

La alternancia en los métodos de elaboración de SPS, desde los primeros teclados de máquinas de escribir, hasta los modernos sistemas de reconocimiento de habla que han modificado los parámetros estéticos del subtitulado, son, en la mayoría de los casos, producto de la tradición práctica adoptada del subtitulado ordinario (cf. Ivarsson y Carroll 1998: 49).

Estrechamente vinculados entre si $i^{3}$, los parámetros "Tipografía" y "Color" han sido fuente recurrente de numerosos estudios en el seno del SPS, desde la traducción (cf. Neves 2005; Martínez-Tejerina 2008) a la comunicación

3. Cuando la aplicación cromática no afecta al conjunto del subtítulo, el empleo del color forma parte del parámetro "Tipografía". 
audiovisual (cf. Carrero y Souto 2008; Utray et al. 2010), pasando por la ingeniería (cf. Kirkland 1999; Martín et al. 2007; Martín et al. 2008). La focalización temática, unida a la desactualización de algunas de las normativas que todavía continúan fundamentándose en la tecnología del teletexto analógico en vías de extinción (cf. AENOR 2003: 3; BCI 2005: 2; ITC 1999: 6), hace que muchos de los parámetros estéticos no se lleguen a abordar de forma explícita en los textos normativos. De este modo, por ejemplo, el sistema analógico europeo justificaba la imposición de un tipo de fuente (teletexto), de unos colores determinados, la utilización de una caja sobre la que se representaban los subtítulos, el espaciado e interlineado de los mismos, etc. (cf. AENOR 2033: 5; BCI 2005: 2); mientras, en los Estados Unidos, el sistema Línea 21 forzaba el uso de mayúsculas y la imposibilidad de emplear combinaciones cromáticas para el SPS (cf. King et al. 1994). No obstante, al margen de la evolución o las limitaciones técnicas y tecnológicas que han perfilado las diferencias estéticas, también es posible encontrar usos diferenciados en otros países, como es el caso de Francia, en el que las combinaciones cromáticas de cajas y subtítulos adoptan una interpretación única a la hora de representar la información extralingüística (cf. Bouzinac 2008: 5).

Para poder entender la gran complejidad que entraña el parámetro "Tipografía" y su relevancia en el SPS, en la tabla que se recoge a continuación

\begin{tabular}{|c|c|c|c|c|c|c|c|c|c|c|c|c|}
\hline & 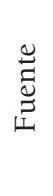 & 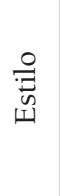 & 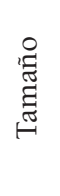 & $\frac{\tilde{0}}{0}$ & 䒕 & 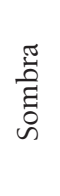 & 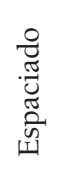 & 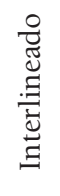 & $\frac{\sqrt[\sigma]{\tilde{J}}}{\cup}$ & 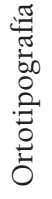 & 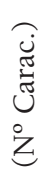 & 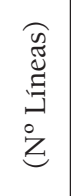 \\
\hline Fuente & & $\mathrm{X}$ & $\mathrm{X}$ & & $\mathrm{X}$ & $\mathrm{X}$ & $\mathrm{X}$ & & & $\mathrm{X}$ & $\mathrm{X}$ & \\
\hline Estilo & $\mathrm{X}$ & & $\mathrm{X}$ & & $\mathrm{X}$ & $\mathrm{X}$ & $\mathrm{X}$ & $\mathrm{X}$ & & $\mathrm{X}$ & $\mathrm{X}$ & \\
\hline Tamaño & $\mathrm{X}$ & $\mathrm{X}$ & & & $\mathrm{X}$ & $\mathrm{X}$ & $\mathrm{X}$ & $\mathrm{X}$ & & & $\mathrm{X}$ & $\mathrm{X}$ \\
\hline Color & & & & & $\mathrm{X}$ & $\mathrm{X}$ & & & $\mathrm{X}$ & & & \\
\hline Borde & $\mathrm{X}$ & $\mathrm{X}$ & $\mathrm{X}$ & $\mathrm{X}$ & & $\mathrm{X}$ & & & $\mathrm{X}$ & & & \\
\hline Sombra & $\mathrm{X}$ & $\mathrm{X}$ & $\mathrm{X}$ & $\mathrm{X}$ & $\mathrm{X}$ & & $\mathrm{X}$ & & $\mathrm{X}$ & & & \\
\hline Espaciado & $\mathrm{X}$ & $\mathrm{X}$ & $\mathrm{X}$ & & & $\mathrm{X}$ & & & & $\mathrm{X}$ & $\mathrm{X}$ & \\
\hline Interlineado & & $\mathrm{X}$ & $\mathrm{X}$ & & & & & & & & & \\
\hline Caja & & & & $\mathrm{X}$ & $\mathrm{X}$ & $\mathrm{X}$ & & & & & & \\
\hline Ortotipografía & $\mathrm{X}$ & $\mathrm{X}$ & & & & & $\mathrm{X}$ & & & & $\mathrm{X}$ & \\
\hline ( $\mathrm{N}^{\circ}$ carac. $)$ & $\mathrm{X}$ & $\mathrm{X}$ & $\mathrm{X}$ & & & & $\mathrm{X}$ & & & $\mathrm{X}$ & $\mathrm{X}$ & $\mathrm{x}$ \\
\hline ( $\mathrm{N}^{\circ}$ líneas) & & & $\mathrm{X}$ & & & & & & & & $\mathrm{X}$ & $\mathrm{x}$ \\
\hline
\end{tabular}

Figura 1 
se representa la interdependencia que se establece entre los sub-parámetros que la integran. Las "X" muestran aquellos sub-parámetros que se verían modificados por otros en función de las variables seleccionadas para cada caso. Así, por ejemplo, la elección de un determinado tipo de fuente podría limitar las opciones de estilo, borde o sombra que se le podrían aplicar a la misma, su tamaño final, el espaciado entre caracteres, la idoneidad de los rasgos ortotipográficos implícitos en ella y el número de caracteres que sería posible representar con la fuente seleccionada en un subtítulo determinado y así sucesivamente.

Pese a todo, de todos los "Parámetros estéticos", es posible que el parámetro "Tipografía" resulte el menos específico del SPS. Sin embargo, teniendo en cuenta la relevancia que tiene la visibilidad y legibilidad del subtítulo para los usuarios con deficiencias auditivas, dado que un alto porcentaje de este grupo de usuarios presenta problemas de discriminación cromática (cf. BBC 2009: 18; Romero-Fresco 2010: 183), resulta de especial importancia su estudio detallado.

Otro de los parámetros no específicos del SPS es el parámetro "Posición", al que, como ya se ha avanzado, se le ha añadido una nueva categoría técnica, la "Justificación", integrada como una variable de posicionamiento (cf. Bartoll 2008: 264). Teniendo en cuenta el tratamiento que se le da en las diferentes normativas, y la práctica generalizada, se ha creído necesario analizar la posición en base a dos desplazamientos del subtítulo en pantalla: vertical (posición) y horizontal (justificación). Como ya se ha planteado al abordar los parámetros extralingüísticos, la modificación del parámetro "Posición" es uno de los elementos más extendidos en SPS. Pese a que la práctica generalizada recoge la representación del subtítulo en una posición inferior centrada (cf. Neves 2005: 201), es habitual que esta práctica se modifique en el caso del SPS para dar cabida a la información extralingüística, debido a la necesidad de identificar a los personajes o de representar efectos sonoros o música (cf. Bartoll y Martínez-Tejerina 2010: 69).

El último de los parámetros de este grupo, el "Emplazamiento", que hace referencia a la ubicación del subtítulo sobre la pantalla de la obra audiovisual, o fuera de ella, no suele ser mencionado en las diferentes normativas disponibles. Estas, que tratan de armonizar el ejercicio del SPS se centran, de manera casi exclusiva, en la televisión, por lo que únicamente abordan la elaboración de subtítulos internos. Su desarrollo dependerá, sin embargo, del tipo de obra audiovisual a la que se vaya a aplicar al SPS. La subtitulación de actos en directo, en la que se suele emplear el subtitulado en emplazamiento externo, es, a menudo, considerada una modalidad de SPS. No obstante, criterios 
pragmáticos (momento de elaboración), técnicos (elaboración) y estético-técnicos (incorporación), limitarían la presencia de parámetros extralingüísticos y estéticos (tipografía y posición) específicos de esta modalidad.

\subsection{Parámetros técnicos}

Los "Parámetros técnicos" planteados en la presente propuesta taxonómica, a diferencia de los recogidos por Bartoll, harían referencia de manera exclusiva a aquellos aspectos de la producción de SPS menos visibles para el espectador. Como sucede con los pragmáticos, los parámetros técnicos apenas plantean diferencias específicas en el caso del SPS. La restringida visibilidad de este grupo de parámetros hace que no resulte extraño que la naturaleza técnica del SPS no suela estar representada de manera explícita en las normativas escritas, ni en los estudios académicos.

Tres son los parámetros definidos en la propuesta original: "Difusión", "Archivado" y "Formato". De todos ellos el "Archivado", que hace referencia a la vinculación física de subtítulo e imagen, y el "Sistema de difusión", que representa la forma de proyección de los subtítulos desde un punto de vista técnico, no plantean diferencias significativas entre las distintas modalidades de subtitulado. Sin embargo, no sucede lo mismo con el "Formato", o código de almacenamiento de los subtítulos, dependiente en gran medida de las características estéticas del SPS. Mientras muchos de los formatos empleados almacenan exclusivamente aspectos lingüísticos y temporales del subtítulo, el SPS precisa en igual medida del almacenamiento de rasgos cromáticos y posicionales esenciales en esta modalidad, algo que solo se consigue con determinados formatos, entre los que actualmente destacan Substation Alpha, Advanced Substation Alpha o Viplay Subtitle File (cf. Bartoll 2008: 268). Dentro de los parámetros técnicos, este es uno de los aspectos de mayor estudio y crecimiento, impulsados por las innovaciones tecnológicas y el surgimiento de nuevos medios.

Sin embargo, es posible añadir dos nuevos parámetros a esta categoría: "Método de elaboración" y "Medio", que hacen referencia al sistema de transcripción de los subtítulos y al soporte al que se incorporan los subtítulos respectivamente. Ambas aportaciones, igualmente aplicables al subtitulado general, condicionan en gran medida las características de cualquier tipo de subtítulo.

De todos los parámetros técnicos, el "Medio" es el más relevante para cualquier modalidad de subtitulado, y en el caso del SPS, requiere una especial consideración si se tiene en cuenta las diferencias estéticas y técnicas que lleva implícitas (cf. Arnáiz-Uzquiza 2007: 37; Romero-Fresco 2011: 38). 
Las diferentes características y posibilidades de cada uno de los soportes audiovisuales (televisión, DVD, Internet, videojuegos, actos en directo, etc.), que apenas han llegado a ser objeto de estudio por parte de los académicos, obligan al subtitulador a valorar este parámetro antes de abordar cualquier otro aspecto, ya sea lingüístico, extralingüístico, pragmático, estético, técnico o estético-técnico.

Por lo que respecta al "Método de elaboración", si bien en la mayoría de los casos estos subtítulos se elaboran utilizando el método de teclado ordinario, para la producción de subtítulos en directo (simultáneos) suele utilizarse otro tipo de teclados (máquinas de estenotipia, velotipia, palantipia) y sistemas que permiten una transcripción más rápida, como es el caso del reconocimiento de habla (cf. Romero-Fresco 2011: 35). Como ya se ha apuntado para el parámetro "Emplazamiento", el tipo de productos audiovisuales para los que se emplean estos métodos no permite hablar de una forma de subtitulación exclusiva para sordos, pues suelen ser materiales en los que no es habitual la presencia de información extralingüística (cf. Vela 2007: 7). Por lo tanto, todavía a día de hoy su demanda funcional suele estar cubierta por modalidades de subtitulado ordinario.

\subsection{Parámetros estético-técnicos}

El último de los grupos de parámetros que se incorporarían a la propuesta de Bartoll estaría a medio camino entre los "Parámetros estéticos" y los "Parámetros técnicos", ya que, aunque el espectador percibe el resultado visual de la manipulación de las variables, no se trata de una elección estética del subtitulador, sino de una consecuencia del proceso de producción y de configuración del material final.

Mientras el parámetro "Incorporación", que hace referencia a la forma en la que el texto escrito aparece en pantalla, está estrechamente ligado al "Método de elaboración" técnico o la "Intención" pragmática, el parámetro "Opcionalidad" (visibilidad obligatoria, o no, de los subtítulos) suele estar marcado por los parámetros "Destinatarios" y "Medio de difusión" (figura 2). Esta focalización en el grupo de usuarios hace que, sin resultar ninguno de los dos aspectos estético-técnicos exclusivos del SPS, sea posible decir que el parámetro "Opcionalidad" está más estrechamente vinculado a esta disciplina audiovisual. Desde que en 1972 aparecieran los primeros ejemplos no opcionales de SPS (cf. DCMP 2010: 7) y urgiese la necesidad de diseñar sistemas para su difusión reduciendo su visibilidad, la opcionalidad ha sido la elección habitual del SPS. Reducida su presencia de forma casi exclusiva a las páginas del teletexto o a los menús de idiomas del DVD, únicamente el desarrollo de 
nuevas tecnologías de distribución, como es el caso de Internet y sus múltiples plataformas, está permitiendo facilitar el acceso a esta herramienta de accesibilidad audiovisual.

Pero al margen de estos dos parámetros, ya identificados en la taxonomía anterior, la incorporación del parámetro "Velocidad" supone la principal aportación dentro de esta categoría a la propuesta de Bartoll. Suele analizarse de manera conjunta con otro parámetro lingüístico importante en esta modalidad, la "Densidad" (a menor densidad, mayor velocidad) y es uno de los elementos de principal importancia en el seno del SPS. Sin embargo, mientras que con el parámetro lingüístico los usuarios reclaman la transcripción literal del texto (cf. OFCOM 2005: 16; Romero-Fresco, en prensa), no son habituales las referencias al parámetro "Velocidad". No obstante, su modificación supondría, por ejemplo, elevar el número de caracteres por segundo y línea y una reducción en los tiempos de exposición, lo que alteraría considerablemente el patrón de lectura (figura 2). Sin resultar exclusivo del SPS, pero esencial para su óptimo desarrollo, es uno de los parámetros que ha concentrado un mayor número de estudios en el seno de esta disciplina en los últimos años (cf. Cambra et al. 2008; Romero-Fresco 2010; Romero-Fresco, en prensa). Las velocidades del SPS propuestas por algunas normativas recogen cifras que suelen ser similares entre sí y se establecen en torno a las 180 palabras por minuto (ppm) como máximo: 140-180 ppm (cf. BBC 2009: 7); 160-180 ppm (cf. BCI 2005: 5); 180 ppm (cf. DFA 2004: 6); 200 ppm (cf. CAB 2008: 21). En España, por ejemplo, donde la UNE-153010 actual (2003) plantea una cifra de 19 caracteres por segundo, o $150 \mathrm{ppm}$, la velocidad es considerablemente superior a los 12 caracteres por segundo propuestos para el subtitulado ordinario en español (cf. Díaz-Cintas 2003: 118). De igual manera, no es posible equiparar totalmente las velocidades de lectura en inglés y español, expresadas en "palabras por minuto", si no se tiene en cuenta que la longitud media de una palabra inglesa es de 4,5 caracteres, mientras en español es de 4,9 (cf. Pierce 1980: 75; Morelli 2010). Ambos planteamientos, unidos a la necesidad de ajustar las velocidades en función de los públicos y sistemas de incorporación de subtítulos (cf. Romero-Fresco, en prensa), hacen que en la actualidad se esté revisando este parámetro en la redacción de la nueva normativa que regule el ejercicio del SPS.

\section{Conexiones entre parámetros}

Como se ha podido comprobar al analizar la tipografía de los subtítulos (figura 1), son muchos los parámetros y sub-parámetros que entran en juego en el desarrollo del SPS y la estrecha relación que se establece entre ellos hace que 
sea preciso tener en cuenta cualquier mínima variación (figura 2). Los 23 parámetros que dan forma a la presente taxonomía y sirven para describir el SPS establecen una compleja serie de relaciones que dan cuenta de la dependencia que se establece entre ellos. De este modo, por ejemplo, la elección de una determinada opción de identificación de personajes tendría implicaciones desde el punto de vista lingüístico (en la densidad, si se adopta la identificación mediante etiquetas), pragmático (en la autoría, en cualquier caso), estético (si se opta por el desplazamiento) o técnico (por restricciones de formato).

La siguiente tabla representa las relaciones de interdependencia que se establecen entre todos los parámetros identificados. Las "X" marcan aquellos parámetros en los cuales la elección de una determinada variable condicionaría la elección de variables en otros parámetros.

Como se puede comprobar, los "Parámetros pragmáticos" y, en especial, el parámetro "Destinatarios", son los que más peso tienen en su conjunto por ser los que justifican el objetivo final del subtítulo. Este será el punto de partida para determinar, en el caso concreto del SPS, que un subtítulo se desarrolla específicamente para públicos con deficiencia auditiva. Pero no es el único parámetro determinante en este sentido. También desde el punto de vista técnico es posible marcar la dependencia. Al igual que en el parámetro "Destinatarios", el parámetro "Medio" condiciona la totalidad de las variables que se aplican al SPS, e incluso aspectos en apariencia no vinculados, como la elección lingüística, resultarían relevantes en determinados contextos (cf. apartado 1.1).

Pero al margen de estos, cabría analizar la especial relevancia de otros grupos de parámetros, como es el caso de los "Parámetros extralingüísticos sonoros". Si se analiza la tabla en detalle, es posible comprobar que aquellos parámetros con mayor peso en el desarrollo del SPS, por el efecto que puede tener su modificación en otros (como sucede con los parámetros pragmáticos y técnicos), no son aquellos exclusivamente representativos de esta modalidad. Sin embargo, la presencia de los parámetros específicos del SPS adquiere especial relevancia al condicionar a todos los demás grupos de parámetros. La incorporación de información extralingüística altera los componentes lingüísticos ("Densidad"), estéticos ("Color", "Tipografía" y "Posición"), técnicos ("Formato") y estético-técnicos ("Velocidad", "Opcionalidad"), dotando al subtítulo de características muy marcadas. No obstante, su representación también se ve modificada por aspectos pragmáticos ("Momento de elaboración") y técnicos ("Método de elaboración" y "Medio"). 


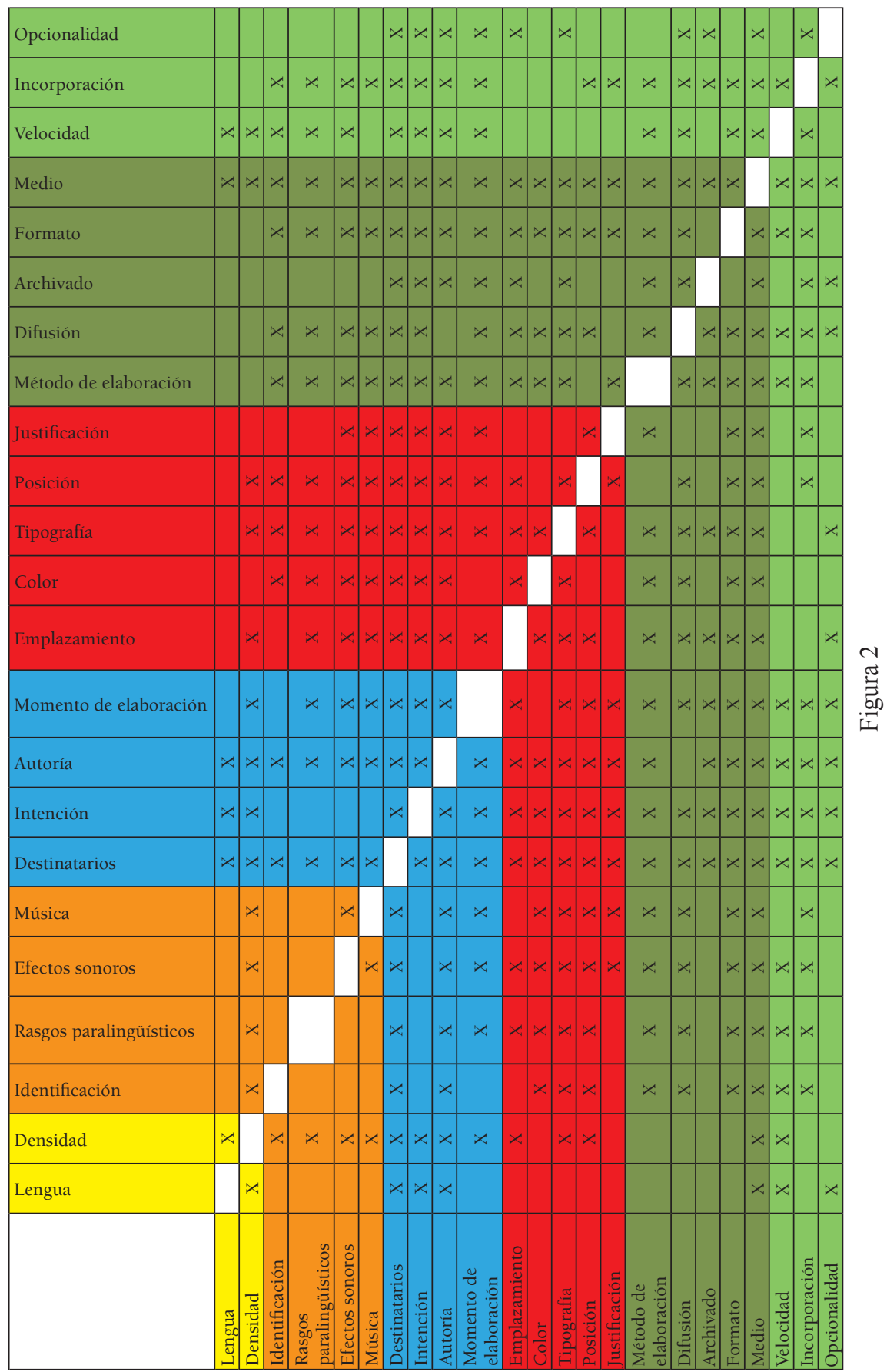




\section{Conclusiones}

Dada la escasa disponibilidad de otras modalidades de accesibilidad audiovisual para las personas con discapacidad auditiva, y la gran heterogeneidad que caracteriza a este colectivo de usuarios, es preciso conocer todos los factores que condicionan el ejercicio del SPS en cada una de sus etapas y su resultado final. Partiendo de la taxonomía desarrollada por Bartoll para el subtitulado ordinario, hemos planteado el desarrollo de una clasificación específica para el estudio del proceso de elaboración del SPS en su totalidad. La necesidad de incorporar elementos específicos de esta modalidad, así como otros elementos y parámetros no exclusivos de la misma, pero de especial relevancia para su desarrollo, justificaría esta iniciativa. De esta forma, la nueva taxonomía estaría integrada por los grupos de parámetros: "Lingüísticos", "Extralingüísticos", "Pragmáticos", "Estéticos", "Técnicos" y "Estético-técnicos".

Desde el punto de vista lingüístico, al margen de la dualidad marcada por la tradición audiovisual de países dobladores y subtituladores, el parámetro "Densidad" es el más relevante. Son muchos los estudios que ya se han llevado a cabo y que han dejado constancia de las diferentes capacidades lectoras de los grupos de usuarios, así como de sus demandas en lo que a literalidad se refiere. Teniendo en cuenta que, en la actualidad, los espectadores con problemas de audición únicamente disponen de una modalidad de SPS, las necesidades del colectivo quedan cubiertas de forma dispar en lo que a aspectos lingüísticos se refiere.

La información extralingüística, exclusiva de esta modalidad de subtitulado, es la principal incorporación a la taxonomía original. Los aspectos extralingüísticos representan toda la información no verbal que resulta preciso recoger para ofrecer toda la información que compone la obra audiovisual al público sordo. Dada la naturaleza variada de este tipo de información, es posible definir hasta cuatro parámetros en este grupo ("Identificación de personajes", "Información paralingüística", "Efectos sonoros" y "Música"), cuya representación altera la composición del subtítulo tradicional. Las variables adoptadas para la representación de cada uno de los parámetros suelen tener su origen en la tradición, por lo que varían de unos países a otros. Esta variabilidad suele ir acompañada de una modificación de otros grupos de parámetros, como son los lingüísticos y los estéticos, dando origen a una sucesión de variaciones en otra serie de parámetros.

A pesar de haber sido objeto de un número menor de estudios académicos dentro del SPS y de estar apenas representados en las normativas publicadas, los parámetros pragmáticos son el conjunto con mayor peso específico en la taxonomía, al tener la capacidad de modificar la gran mayoría de los 
parámetros de la misma. Estos parámetros marcan la intencionalidad y funcionalidad de los subtítulos, por lo que gran parte de la efectividad de los mismos radicaría aquí. La aplicación efectiva de los parámetros pragmáticos ha de servir para desarrollar materiales totalmente adaptados a las necesidades de cada grupo de usuarios y, entre ellos, los usuarios con problemas de audición.

Los "Parámetros estéticos" representan los aspectos visuales del subtítulo, y constituyen el grupo de parámetros de mayor relevancia en términos de legibilidad, directamente relacionado con la percepción y la comprensión. Son muchos los parámetros integrados en este grupo. A pesar de no resultar exclusivos del SPS, la representación de la información extralingüística hace que determinados aspectos ("Color", "Tipografía" o "Posición") se vean modificados de forma más o menos directa.

Los "Parámetros técnicos", por el contrario, se centran en la elaboración y diseminación del SPS. En lugar de verse condicionados, este grupo de parámetros suele condicionar la inclusión de información extralingüística. El "Método de elaboración" empleado o el "Formato", permitirán o no la inclusión de esta información exclusiva del SPS, siendo el "Medio" el parámetro más trascendental en este sentido.

A medio camino entre los dos grupos de parámetros anteriores, los "Parámetros estético-técnicos" recogen los aspectos cuya producción tiene un reflejo visual. El más representativo de este grupo, el parámetro "Velocidad", se ha convertido en el aspecto más analizado en los últimos tiempos desde esta disciplina, por la aparición de nuevos "Métodos de elaboración" que ayudarían a incrementar la literalidad demandada por los espectadores.

La suma de las 6 categorías que agrupan los 23 parámetros de la nueva taxonomía dibuja un panorama detallado del proceso de producción del SPS. La identificación de un grupo de parámetros específico de esta modalidad de subtitulado ("Parámetros extralingüísticos sonoros"), y las consecuencias que su incorporación conlleva, no logran imponerse, sin embargo, al peso de otros parámetros considerados generales ("Parámetros pragmáticos" y "Parámetros técnicos"). Los estudios que en los últimos años han surgido impulsados por el creciente avance del SPS en el mercado han tratado de abordar el análisis de aspectos especialmente representativos para su desarrollo, como es el caso de la densidad, la posición o la tipografía entre otros. Sin embargo, el hecho de tratarse de iniciativas centradas en parámetros aislados hace que, en la mayoría de las ocasiones, no se tenga en cuenta la interdependencia entre parámetros y las consecuencias que cualquier cambio implicaría para otras variables.

Esta taxonomía pretende ser no solo una herramienta para el análisis integral del proceso de subtitulado para sordos, sino también un instrumento 
de reflexión sobre las consecuencias del estudio aislado de cada uno de los parámetros que configuran la práctica del SPS.

\section{Bibliografía}

ARnÁIz UzQUizA, Verónica. (2007) El subtitulado para sordos en España. Trabajo fin de máster. Barcelona: Universitat Autònoma de Barcelona.

BARTOLL, Eduard. (2008) Paramètres per a una taxonomia de la subtitulació. Tesis doctoral inédita. Universitat Pompeu Fabra. Versión electrónica: <http://hdl. handle.net/10803/7572>

BARTOLL, Eduard \& Anjana Martínez-Tejerina. (2010) "The positioning of subtitles for the deaf and hard of hearing". En: Matamala, Anna \& Pilar Orero (eds.) 2010. Listening to Subtitles: Subtitles for the Deaf and Hard of Hearing. Berna: Peter Lang. pp. 69-86.

Bouzinac, Alexis. (2008) "Sous-titrage: Lhistorique". Media Sous titres 30. Versión electrónica: <http://www.tentatives-accessibles.eu/share/sous-titragetelevision.pdf>

CAmbra, Cristina; Nuria Silvestre \& Aurora Leal. (2008) "Función de la subtitulación de los dibujos animados". Comunicar 32. pp. 32-36.

CArrero, Juan Manuel \& Mónica Souto. (2008) "Guía de buenas prácticas para el subtitulado para sordos en DVD”. En: Jiménez, Catalina \& Ana Rodríguez (eds.) 2008. Accesibilidad a los medios audiovisuales para personas con discapacidad. Amadis 2007. Madrid: Real Patronato sobre Discapacidad. pp. 89-100.

CiverA, Clara \& Pilar Orero. (2010) "Introducing icons in subtitles for the deaf and hard of hearing: Optimising reception?" En: Matamala, Anna \& Pilar Orero (eds.) 2010. Listening to Subtitles: Subtitles for the Deaf and Hard of Hearing. Berna: Peter Lang. pp. 149-162.

DE LINDE, Zoe. (1996) "Le sous titrage intralinguistique pour les sourds et les malentendants". En: Gambier, Yves (ed.) 1996. Les transferts linguistiques dans les medias audiovisuelles. Paris: Presses Universitaires du Septentrion. pp. 165-183.

De Linde, Zoe \& Neil Kayl. (1999) The semiotics of subtitling. Manchester: St. Jerome.

Described and Captioned Media Program (DCMP). (2010) How bird hunting saved captioning? Versión electrónica: <http://www.dcmp.org/caai/nadh36. $\mathrm{pdf}>$

DíAz Cintas, Jorge. (2003) Teoría y práctica de la subtitulación (inglés-español). Barcelona: Ariel Cine.

FELS, Deborah I.; Caniel Lee; Carmen Branje \& Matthew Hornburg. (2005) "Emotive captioning and access to television". AMCIS 2005 Proceedings. Versión electrónica: <http://aisel.aisnet.org/amcis2005/300> 
GotTlieb, Henrik. (1997) Subtitles, Translation and Idioms. Tesis doctoral. Copenhague: University of Copenhagen.

Gottlieb, Henrik. (2005) "Multidimensional translation: Semantics turned semiotics". MuTra 2005: Challenges of Multidimensional Translation: Conference Proceedings. Versión electrónica: <http://www.euroconferences.info/ proceedings/2005_Proceedings/2005_Gottlieb_Henrik.pdf>

IVARSSON, Jan \& Mary Carroll. (1998) Subtitling. Simrishamn: TransEdit.

Jensema, Carl; Sameh Sharkawy; Ramalinga Sarma Danturthi; Robert Burch \& David Hsu. (2000) "Eye movement patterns of captioned television viewers". American Annals of the Deaf 145:3. pp. 275-285.

KARAMitroglou, Fotios. (1997) "A proposed set of subtitling standards in Europe”. Translation Journal 2:2. Versión electrónica: <http://translationjournal. net/journal/04stndrd.htm>

KING, Cynthia; Carol LaSasso \& Doug Short. (1994) "Digital captioning: Effects of color coding and placement in synchronized text-audio presentations". En: Maurer, Hermann (ed.) 1994. Educational Multimedia and Hypermedia. Charlottesville: Association for the Advancement of Computing in Education. pp. 329-334.

KIRKLAND, C. Eric. (1999) "Evaluation of captioning features to inform development of digital television captioning capabilities". American Annals of the Deaf 144:3. pp. 250-260.

LlOMBART, Carles. (2007) Tinc un/a alumne/a sord/a a l'aula, Barcelona: Consorci d'Educació de Barcelona.

LONHEIM, Sylfest. (1995) "Lécriture sur l'écran: stratégies de sous-titrage à NRK, une étude de cas". Translatio. Nouvelles de la FIT/FIT Newsletter XIV (3-4). pp. 288-293.

LORENZO, Lourdes. (2010a) "Subtitling for deaf and hard of hearing children in Spain. A case study". En: Matamala, Anna \& Pilar Orero (eds.) 2010. Listening to Subtitles: Subtitles for the Deaf and Hard of Hearing. Berna: Peter Lang. pp. 115-138.

LORENZO, Lourdes. (2010b) "Criteria for elaborating subtitles for deaf and hard of hearing children in Spain: A guide of good practice". En: Matamala, Anna \& Pilar Orero (eds.) 2010. Listening to Subtitles: Subtitles for the Deaf and Hard of Hearing. Berna: Peter Lang. pp. 139-148.

LORENZO, Lourdes. (2001) "Características diferenciales de la traducción audiovisual II. El papel del traductor de subtítulos”. En: Lorenzo, Lourdes \& Ana M. Pereira (eds.) 2001. Traducción subordinada (II): El subtitulado (ingles-español ( galego). Vigo: Servicio de Publicacións da Universidade de Vigo. pp. 11-17.

MARTín, Carlos Alberto; José María Merchán; David Jiménez; José Manuel Menéndez \& Guillermo Cisneros. (2008) "Accesibilidad a la televisión digital interactiva”. En: Jiménez, Catalina \& Ana Rodríguez (eds.) 2008. Accesibilidad a 
los medios audiovisuales para personas con discapacidad. AMADIS '07. Madrid: Real Patronato sobre Discapacidad. pp. 67-77.

MARTín, Carlos Alberto; David Jiménez; Guillermo Cisneros \& José Manuel Menéndez. (2007) "Implantación de la accesibilidad en la televisión digital: situación actual y futuros desarrollos". En: Ruiz, Belén \& Francisco Utray (eds.) 2007. Accesibilidad a los medios audiovisuales para personas con discapacidad. AMADIS '06. Madrid: Real Patronato sobre Discapacidad. pp. 115-129.

MARTínEZ-TEJERINA, Anjana. (2008) "El tamaño importa y la posición también. Estudio piloto sobre el formato de los subtítulos para sordos”. En: PérezUgena, Álvaro \& Ricardo Vizcaíno-Laorga (eds.) 2008. ULISES: Hacia el desarrollo de tecnologías comunicativas para la igualdad de oportunidades: Retos y perspectivas para sordos signantes. Madrid: Observatorio de las Realidades Sociales y de la Comunicación. pp. 85-92.

Morelli, Ralph. (2010) Percentages of Letter Frequencies per 1000 words. Hatford: Trinity College. Versión electrónica: < http://www.cs.trincoll.edu/ crypto/ resources/LetFreq.html>

NeVES, Josélia. (2005) Audiovisual Translation: Subtitling for the Deaf and Hard-ofHearing. Tesis doctoral inédita. Londres: University of Surrey-Roehampton. Versión electrónica: <http://roehampton.openrepository.com/roehampton/ bitstream/10142/12580/1/neves\%20audiovisual.pdf>

NEVES, Josélia. (2009) "Interlingual subtitling for the Deaf and Hard-of-Hearing". En: Díaz-Cintas, Jorge \& Anderman, Gunilla (eds.) 2009. Audiovisual Translation: Language Transfer on Screen. Basingstoke: PalGrave McMillan. pp. 151-169.

Office of Communications (OFCOM). (2005) Subtitling: An issue of speed? Versión electrónica: <http://stakeholders.ofcom.org.uk/binaries/research/ tv-research/subt.pdf>

Pereira, Ana María. (2005) "El subtitulado para sordos: estados de la cuestión en España”. Quaderns. Revista de traducció 12. pp. 161-172. Versión electrónica: <http://ddd.uab.es/pub/quaderns/11385790n12p161.pdf >

PereirA, Ana María. (2010) "Criteria for elaborating subtitles for deaf and hard of hearing adults in Spain: Description of a case study". En: Matamala, Ana \& Pilar Orero (eds.) 2010. Listening to Subtitles: Subtitles for the Deaf and Hard of Hearing. Berna: Peter Lang. pp. 87-102.

PereirA, Ana María \& Lourdes Lorenzo. (2005) "Evaluamos la norma UNE153010. Subtitulado para personas sordas y personas con discapacidad auditiva. Subtitulado a través del teletexto". Puentes 6. pp. 21-26.

PÉREZ-UgenA, Álvaro; Ricardo Vizcaíno-Laorga \& Deborah Rolph. (2010) "Subtitles for the deaf and hard of hearing within a virtual avatar environment: ULISES”. En: Matamala, Anna \& Pilar Orero (eds.) 2010. Listening to Subtitles: Subtitles for the Deaf and Hard of Hearing. Berna: Peter Lang. pp. 209-218. 
Pierce, John Robinson. (1980) An Introduction to Information Theory: Symbols, Signals and Noise. Londres: Constable \& Company.

POYATOS, Fernando. (1994a) La comunicación no verbal I. Madrid: Istmo.

POYATOS, Fernando. (1994b) La comunicación no verbal II. Madrid: Istmo.

QUOC, V.Vy \& Deborah Fels. (2009) "Using avatars for improving speaker identification in captioning”. En: Gross, Tom; Jan Gulliksen; Paula Kotzé; Lars Oestreicher; Philippe Palanque \& Raquel Oliveira (eds.) 2009. INTERACT 2009. International Federation of Information Processing. Part II. Berlin: Springer-Verlag. pp. 916-919

QUOC, V.Vy \& Deborah Fels. (2010) "Using placement and name speaker identification in captioning”. En: Miesenberger, Klaus; Joachim Klaus; Wolfgang Zagler \& Arthur Jarshmer (eds.) 2010. ICCHP, Part II. Berlin: Springer-Verlag. pp. 247-254.

Real Academia Española. (2001) "Extralingüístico". En: Diccionario de la Lengua Española $22^{a}$ ed. Versión electrónica: <http://buscon.rae.es/drael/SrvltGUIBu sUsual?LEMA=extraling\%C3\%BC\%C3\%ADstica\&origen=RAE>

Romero-Fresco, Pablo. (2010) "D'Artagnan and the Seven Musketeers: SUBSORDIG travels to Europe". En: Matamala, Anna \& Pilar Orero (eds.) 2010. Listening to Subtitles: Subtitles for the Deaf and Hard of Hearing. Berna: Peter Lang. pp. 175-190.

Romero-FresCO, Pablo. (2011) Subtitling through speech recognition: Respeaking. Manchester: St. Jerome.

Romero-Fresco, Pablo (ed.) (en prensa) The Reception of Subtitles for the Deaf in Europe. Berlin: Peter Lang.

Utray, Francisco; Belén Ruiz \& José Antonio Moreiro. (2010) "Maximum font size for subtitles in Standard Definition Digital Television: Tests for a font magnifying application”. En: Matamala, Anna \& Pilar Orero (eds.) 2010. Listening to Subtitles: Subtitles for the Deaf and Hard of Hearing. Berna: PeterLang. pp. 59-68.

VELA, Jennifer. (2007) "Análisis comparativo del software de subtitulado y estudio de compatibilidad con la norma UNE 153010:2005". En: II Congreso de Accesibilidad a los Medios Audiovisuales para Personas con Discapacidad: Amadis '07. Granada. Versión electrónica: <http://www.cesya.es/estaticas/ AMADIS07/Ponencias/Ponencias/Ponencia_JenniferVela.pdf>

Weber, Nina. (2010) The Acoustic Channel: Conveying sound, tone, mood and music in SDH across the British audiovisual media. Londres: Roehampton University. Proyecto fin de máster inédito.

Woll, Bencie. (1991) Sign Language on Television. Bristol: CDS, University of Bristol. 
ZÁrATE, Soledad. (2010) "Subtitling for deaf children". En: Bogucki, Łukasz \& Krzysztof Kredens (eds.) 2010. Perspectives on Audiovisual Translation. Lódz Studies in Language, 20. Frankfurt am Main: Peter Lang. pp. 109-122.

Normas de SPS

Asociación Española de Normalización y Certificación (AENOR). (2003) Norma Española UNE 153010. Subtitulado para personas sordas y personas con discapacidad auditiva. Subtitulado a través del teletexto. Madrid: AENOR.

British Broadcasting Corporation (BBC). (2009) Online Subtitling Editorial Guidelines. Versión electrónica: <http://www.bbc.co.uk/guidelines/futuremedia/ accessibility/subtitling_guides/online_sub_editorial_guidelines_vs1_1.pdf>

Broadcasting Commission of Ireland (BCI). (2005) BCI Guidelines: Subtitling. Versión electrónica: <www.bai.ie/pdfs/access_sub_guidelines.pdf>

Canadian Association of Broadcasters (CAB). (2008) Closed Captioning Standards and Protocol for Canadian English Language Television Programming Services. Versión electrónica: <http://www.cab-acr.ca/english/social/captioning/ captioning.pdf>

Deafness Forum of Australia (DFA). (2004) Captioning Quality Code of Practice. Versión electrónica: <http://www.deafnessforum.org.au/pdf/Position\%20 Statements/Captioning\%20Quality\%20V2.pdf>

Described and Captioned Media Program (DCMP). (2011) Captioning Key: Guidelines and Preferred Techniques. Versión electrónica: <http://www.dcmp. org/captioningkey/captioning-key.pdf>

Independent Television Commission (ITC). (1999) Guidance on Standards for Subtitling. Ofcom. Versión electrónica: <http://www.ofcom.org.uk/static/ archive/itc/uploads/ITC_Guidance_on_Standards_for_Subtitling.doc> 


\section{BIONOTE / NOTA BIOGRÁFICA}

\section{Verónica Arnáiz Uzquiza}

BA in Translation and Interpreting from the University of Valladolid (UVA), Spain, and MA in Specialised Translation from the UVA and in Audiovisual Translation from the Universitat Autònoma de Barcelona (UAB). She is currently employed as a lecturer in Translation, Subtitling and Subtitling for the Deaf and the Hard-of-Hearing (UVA). She has also collaborated with the Master in Professional and Institutional Translation (UVA) and the European Master in Audiovisual Translation (UAB). She has published several articles and reviews in different journals and volumes, and has presented a number of papers at international conferences dealing with Translation and Accessibility. She is currently working on her $\mathrm{PhD}$ in the evaluation of $\mathrm{SDH}$ parameters at the Universitat Autonoma de Barcelona. She is a member of the research group CAIAC-Transmedia Catalonia.

Diplômée en Traduction et Interprétation à l'Université de Valladolid (UVA), Master en Traduction de Langages d'Especialité (UVA) et en Traduction Audiovisuelle à l'Universitat Autònoma de Barcelona (UAB). Professeur à l'UVA, responsable des cours de Traduction, sous-titrage et sous-titrage pour les sourds et malentendants. Enseignante aussi dans le Master en Traduction Professionnelle et Institutionnelle (UVA) et le Master Européen en Traduction Audiovisuelle (UAB). Elle a publié plusieurs articles et participé à de nombreuses conférences internationales sur Traduction et Accessibilité. Dans l'actualité, elle est en train de rédiger sa thèse de doctorat sur l'évaluation des paramètres du sous-titrage pour les sourds et malentendants, à l'Universitat Autònoma de Barcelona. Elle est membre du groupe de recherche CAIACTransmedia Catalonia. 
Anexo I

\begin{tabular}{|c|c|c|}
\hline [LINGÜÍsTICO] & [LINGÜÍSTICO] & [EXTRALINGÜÍSTICO] * \\
\hline $\begin{array}{l}\text { LENGUAJE } \\
\text { - Interlingüísticos } \\
\text { - Intralingüísticos }\end{array}$ & $\begin{array}{l}\text { DENSIDAD [*] } \\
\text { - Íntegros (literales) } \\
\text { - Reducidos } \\
\text { - Sub. ordinarios } \\
\text { - Sub. simplificados }\end{array}$ & $\begin{array}{l}\text { IDENTIFICACIÓN DE } \\
\text { PERSONAJES } \\
\text { - Posición } \\
\text { - Etiquetas } \\
\text { - Colores } \\
\text { - Puntuación } \\
\text { - Combinados } \\
\text { - Posición y colores } \\
\text { - Posición y puntuación } \\
\text { - Colores y puntuación } \\
\text { - Sin representación } \\
\text { - Nuevas propuestas + }\end{array}$ \\
\hline $\begin{array}{l}\text { [EXTRALINGÜÍSTICO] * } \\
\text { RASGOS } \\
\text { PARALINGÜÍSTICOS } \\
\text { - Descripción } \\
\text { - Onomatopeyas } \\
\text { - Emoticonos } \\
\text { - Sin representación } \\
\text { - Nuevas propuestas + }\end{array}$ & $\begin{array}{l}\text { [EXTRALINGÜÍSTICO] * } \\
\text { EFECTOS SONOROS } \\
\text { - Descripción } \\
\text { - Onomatopeyas } \\
\text { - Iconos } \\
\text { - Sin representación } \\
\text { - Nuevas propuestas + }\end{array}$ & $\begin{array}{l}\text { [EXTRALINGÜÍSTICO] * } \\
\text { MÚSICA } \\
\text { - Diegética } \\
\text { - Título } \\
\text { - Letra } \\
\text { - Descripción } \\
\text { - Combinados } \\
\text { - Título y letra } \\
\text { - Descripción y letra } \\
\text { - Sin representación } \\
\text { - Nuevas propuestas + } \\
\text { - Extradiegética } \\
\text { - Título } \\
\text { - Letra } \\
\text { - Descripción } \\
\text { - Combinados } \\
\text { - Título y letra } \\
\text { - Descripción y letra } \\
\text { - Sin representación } \\
\text { - Nuevas propuestas + }\end{array}$ \\
\hline
\end{tabular}




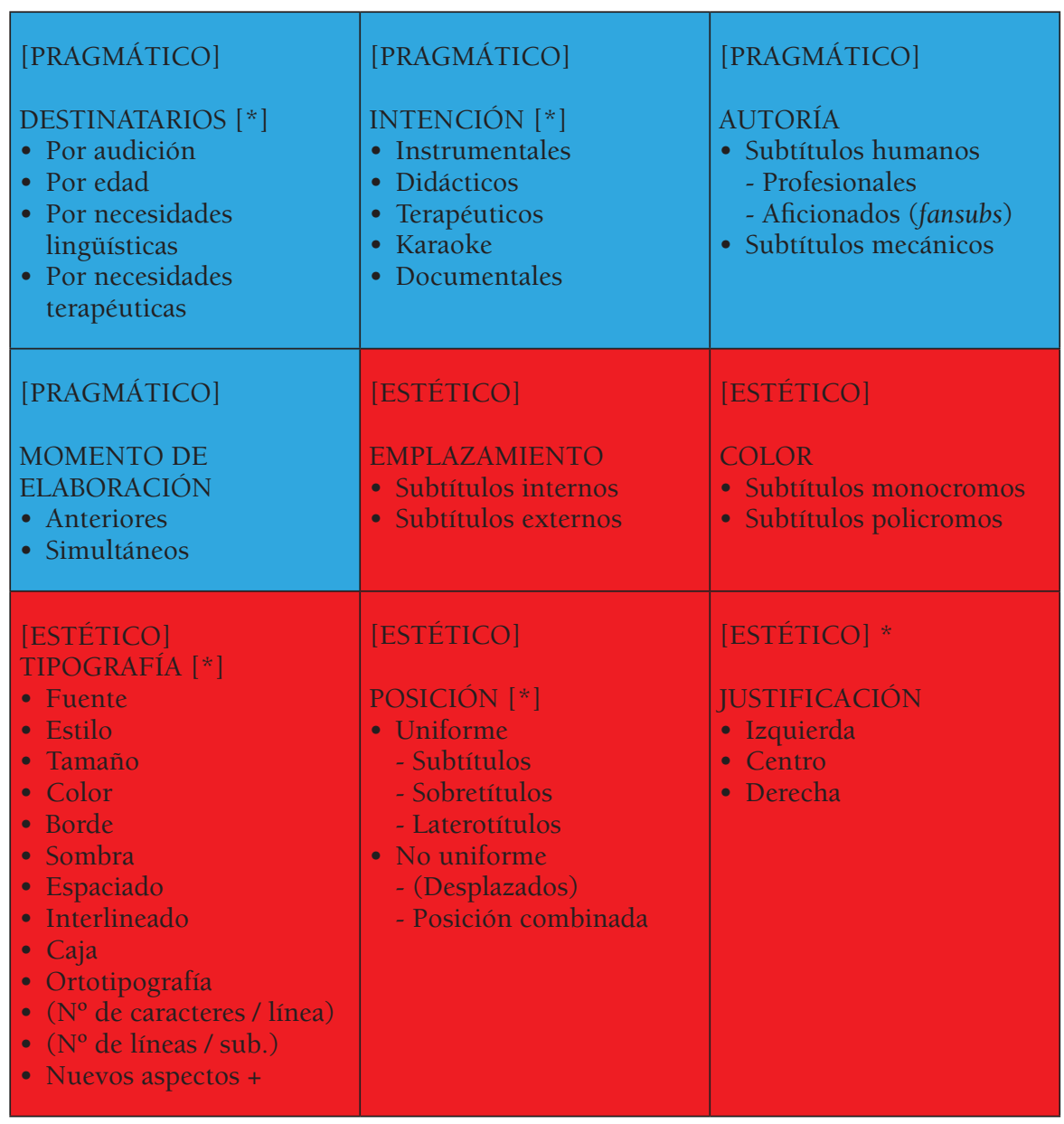




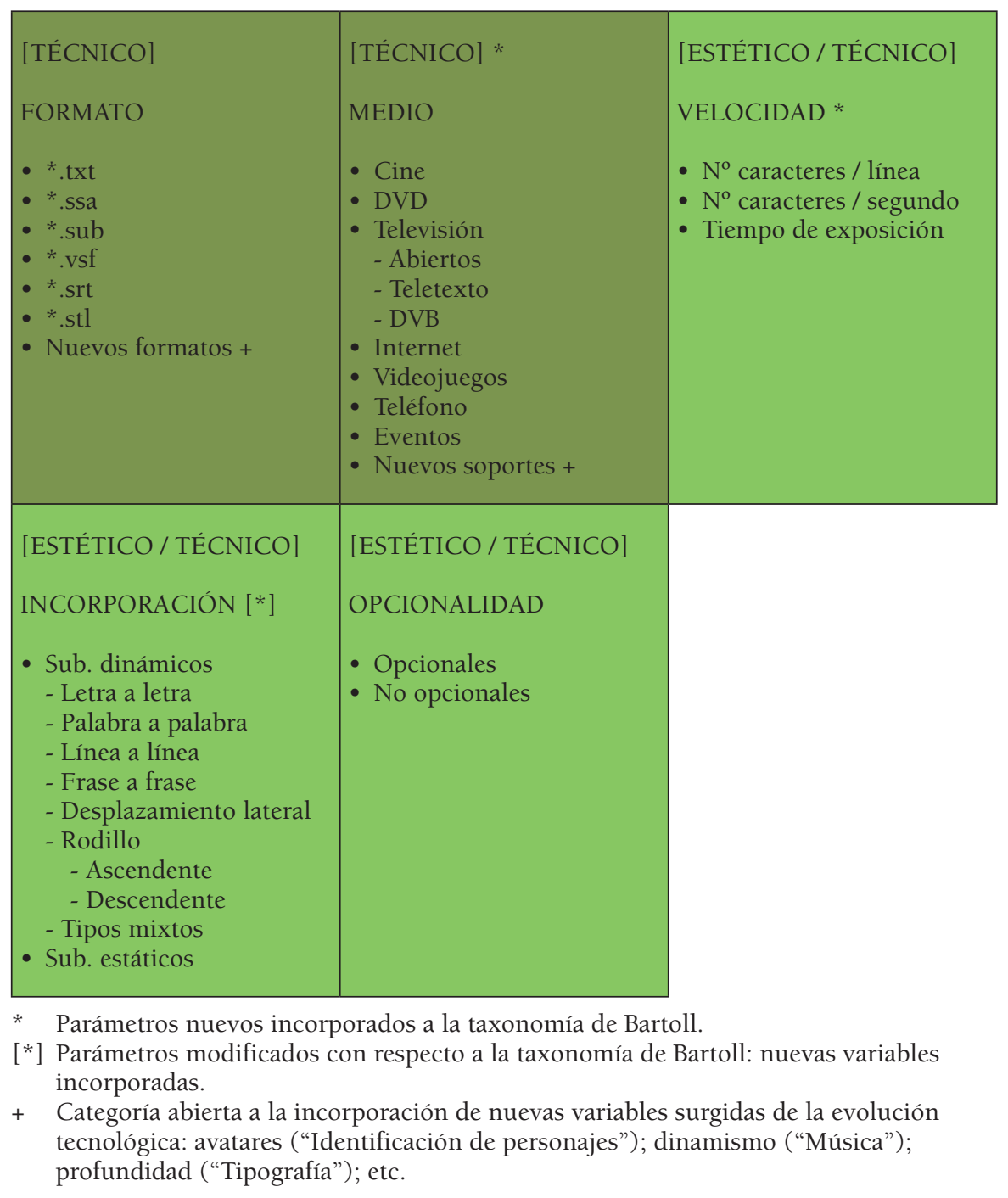

